\title{
Argatroban in Thrombotic Stroke
}

\author{
Marian P. LaMonte \\ Division of Emergency Medicine, University of Maryland School of Medicine, The Maryland Brain Attack Center, \\ Baltimore, Md., USA
}

\section{Key Words}

Stroke $\cdot$ Cerebral blood flow $\cdot$ Cerebral ischemia . Thrombin · Argatroban

\begin{abstract}
Evidence for the potential use of direct thrombin inhibition in the treatment of acute stroke is reviewed. Reduction of secondary microthrombi and improved regional collateral cerebral blood flow is the proposed mechanism of action of thrombin inhibition for the treatment of cerebral ischemia. A clinical study in Japan found that argatroban administered within $48 \mathrm{~h}$ of stroke symptom onset is safe and effective in reducing neurological impairment due to ischemic stroke. The implications of further clinical studies are discussed.
\end{abstract}

Copyright $@ 2002$ S. Karger AG, Basel

\section{Introduction}

The common pathway in precerebral and cerebral arterial occlusion is the fibrin-rich platelet thromboembolism. There are two mechanisms speculated to be important in improving cerebral blood flow in the peri-ischemic penumbra in thromboembolic occlusion. The first targets the cellular aspect of clot formation by reduction in further platelet aggregation, and the second targets alter-

\section{KARGER}

Fax + 41613061234

E-Mail karger@karger.ch

www.karger.com
(C) 2002 S. Karger AG, Basel

Accessible online at:

www. karger.com/pht
1424-8832/02/0329-0039\$18.50/0 ations in the balance of coagulation proteins by accelerating plasminogen conversion or inhibiting thrombin. Drug therapies such as aspirin, clopidogrel or a glycoprotein (GP) IIb/IIIa receptor antagonist such as abciximab are used to interfere with platelet aggregation and agents such as tPA and heparins are used to alter coagulation proteins. Argatroban is a selective and potent antithrombin, which directly blocks thrombin activity without the use of cofactor antithrombin. It also inhibits fibrin formation, platelet aggregation and vascular contraction. Because of these properties, argatroban may participate in both of these mechanisms to achieve the enhancement of cerebral collateral blood flow.

\section{Prior Studies of Anticoagulation Therapy in Acute Cerebral Ischemia}

A recent Cochrane review of anticoagulant therapy started within 2 weeks of ischemic stroke onset included 21 trials involving 23,427 patients [1]. Studies reviewed by the Cochrane collaborative included those in which multiple types of anticoagulants were tested including standard unfractionated heparin, low molecular weight (LMW) heparins, heparinoids, oral anticoagulants, and thrombin inhibitors. Results were disappointing in that there was no evidence that anticoagulation therapy reduced the odds of all-cause mortality or the odds of death
Marian P. LaMonte, MD, MSN, Associate Professor of Neurology and Surgery

Division of Emergency Medicine, University of Maryland Medical Center

Department of Neurology, Room N4W46, 22 South Greene Street

Baltimore, MD 21201 (USA)

Tel. +1 410328 6484, Fax +1 410328 5899, E-Mail mlamonte@umm.edu 
Fig. 1. Relationship between the D dimer and tissue factor levels in the acute phase of ischemic stroke. The values remain within twice that of baseline in argatroban-treated patients. From Kario et al. [7].

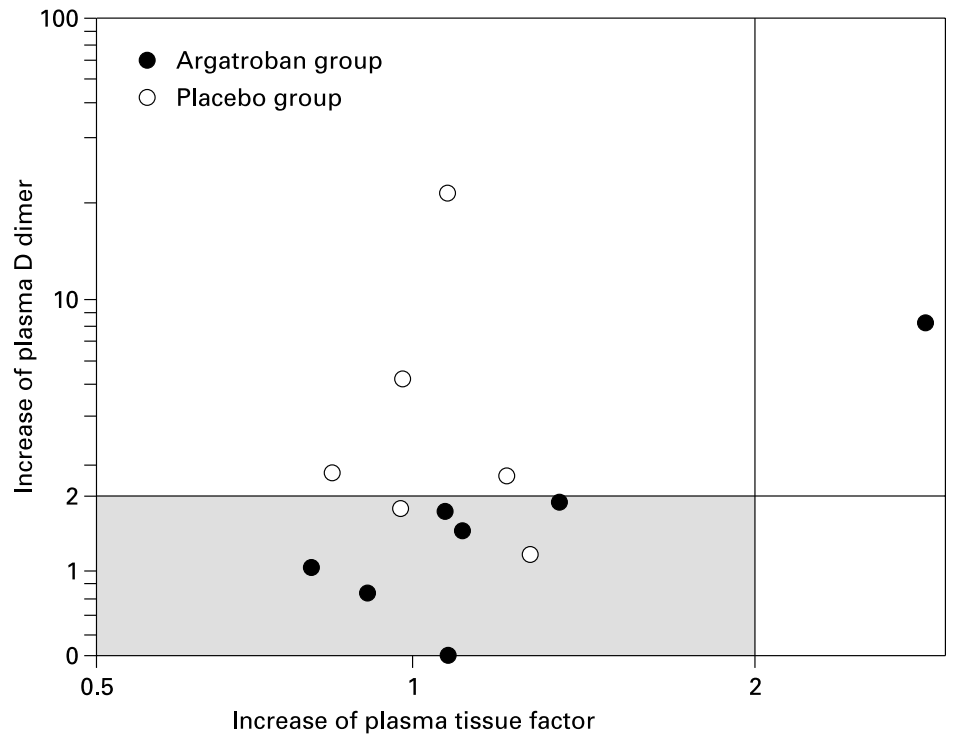

Table 1. Use of markers of platelet activation, coagulation and fibrinolysis in three classifications of acute ischemic stroke (from Uchiyama et al. [6])

\begin{tabular}{|c|c|c|c|}
\hline Stroke type & $\begin{array}{l}\text { Platelet } \\
\text { activation }\end{array}$ & Coagulation & Fibrinolysis \\
\hline \multicolumn{4}{|l|}{ Atherothrombotic } \\
\hline Patients & + & + & + \\
\hline Healthy controls & + & _- & _- \\
\hline Cardioembolic & + & + & + \\
\hline Lacunar & - & - & - \\
\hline
\end{tabular}

or dependency from stroke. In addition, the benefit of 9 fewer recurrent strokes per thousand was offset by an equal number of symptomatic intracranial hemorrhages. Similarly, a combined analysis of the CAST [2] and IST [3] trials concluded that the small gain in prevention of recurrent stroke was countered by the excess risk of intracranial hemorrhage in the anticoagulant-treated group [4]. The CAST and IST trials used aspirin and/or standard unfractionated heparin therapy. Because of a possible lower associated risk of hemorrhage with newer anticoagulants, a follow-up Cochrane review provided information on LMW heparins or heparinoids versus standard unfractionated heparin for acute ischemic stroke [5]. Five trials involving 705 patients treated within 2 weeks of ischemic stroke onset were included in this review. A significant benefit of prevention of deep vein thrombosis accrued to patients treated with LMW heparin or heparinoids as compared to those treated with unfractionated heparin. The number of major bleeding events was too small to provide a reliable estimate of risk, and no information was provided on recurrent stroke or functional outcome to provide a reliable estimate of benefit.

The newer generation anticoagulants, including synthetic direct-acting thrombin inhibitors, such as argatroban, and GP IIb/IIIa and factor Xa inhibitors, offer theoretical advantages over LMW and unfractionated heparins due to their more targeted inhibition of the coagulation cascade. Several clinical studies have been performed to explore the state of coagulation and fibrinolytic changes during ischemic stroke to advance the use of these more targeted therapies.

\section{Studies of Coagulation and Fibrinolysis in Cerebral Ischemia}

Using platelet activation, coagulation and fibrinolysis markers, Uchiyama et al. [6] attempted to profile such changes within specific ischemic stroke subtypes. Stroke types were classified according to the National Institute of 
Fig. 2. Action of argatroban on incidence of microthrombi in rat brain. a Formation of secondary microthrombi in controls. b Formation of secondary microthrombi in argatroban $(0.3 \mathrm{mg} / \mathrm{h} / \mathrm{rat})$-treated rats. From Kawai et al. [8].

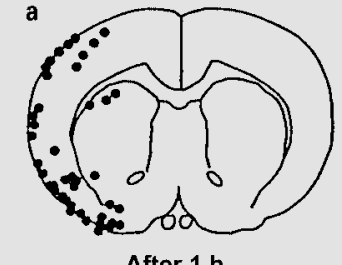

After $1 \mathrm{~h}$

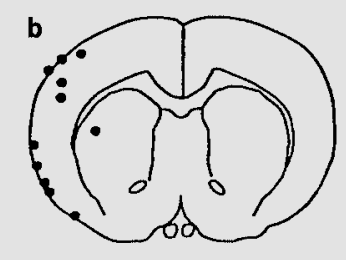

After $1 \mathrm{~h}$

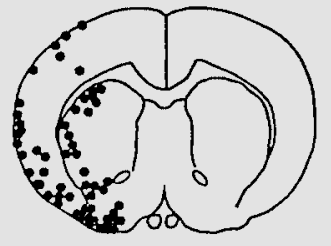

After $3 \mathbf{h}$

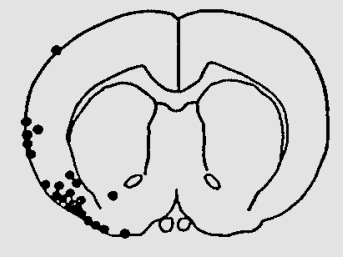

After $3 \mathrm{~h}$

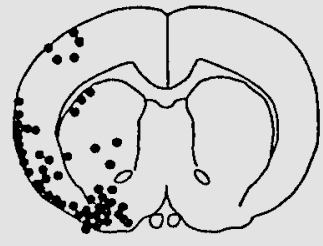

After $6 \mathrm{~h}$

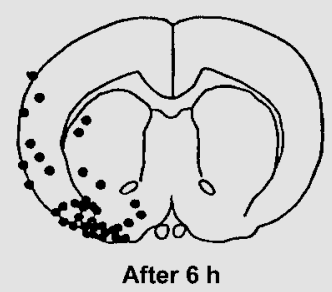

Neurological Disorders and Stroke (NINDS) Stroke Data Bank and included cardioembolic, atherothrombotic (separated into clinically stable or progressive subtypes), and lacunar. Clinical and laboratory testing of patients was performed to determine the category of cerebral infarction. Platelet function tests carried out were platelet aggregation, platelet activating factors (PAF), shear stress, platelet specific proteins, e.g. $\beta$-thromboglobulin and platelet factor 4 (PF4), platelet survival, lysis, accumulation, fibrinogen binding to platelet membrane and expression of P-selectin on platelet surfaces. Coagulation tests employed were antithrombin III, thrombin-antithrombin III complex (TAT) and fibrinopeptide A (FPA). Fibrinolysis markers were $\mathrm{D}$ dimer and plasma- $\alpha_{2}$-plasmin inhibitor complex. All markers were assessed 7 days after onset of stroke.

Their study showed that marked platelet activation was seen in patients with either progressive or nonprogressive atherothrombotic strokes.

However, in patients with progressive strokes, coagulation and fibrinolysis were also observed.

Patients with cardioembolic strokes demonstrated marked fibrinolysis as well as platelet activation. Neither of these was seen in lacunar stroke possibly because there is not enough tissue damage in this stroke subtype (table 1).

Another study assessed D dimer and tissue factor levels as coagulation markers in acute ischemic stroke patients treated with either argatroban or placebo (fig. 1) [7]. Compared with baseline, the changes in values, 7 days after the acute episode, were less in the argatroban-treated patients and remained within twice the values on day 1 .

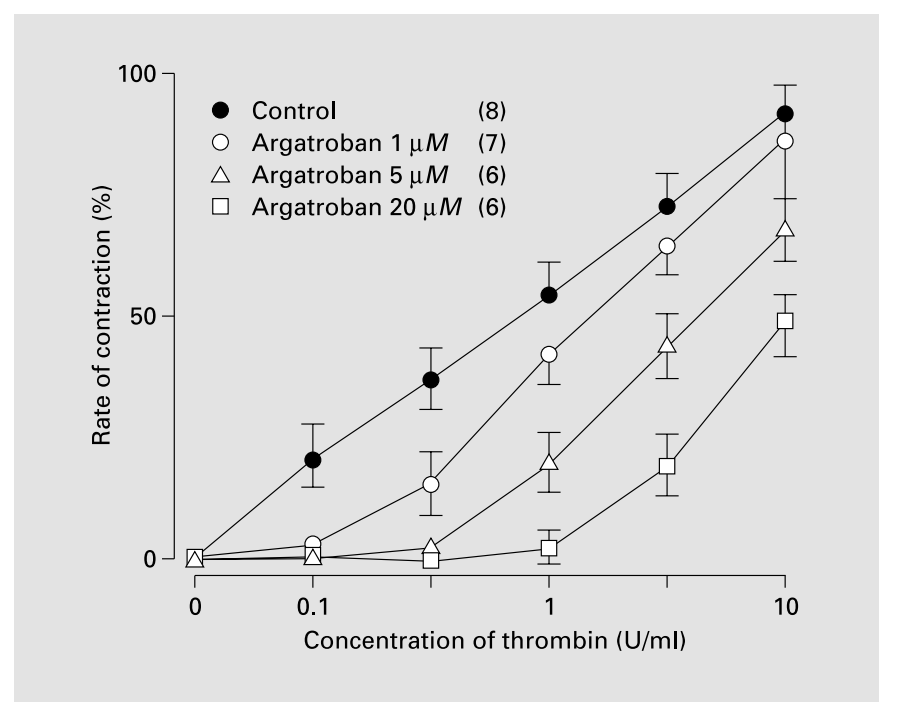

Fig. 3. Inhibitory effect of argatroban on dog basilar artery contraction.

\section{Argatroban in Animal Models}

The potential for argatroban to block microthrombus formation and artery smooth muscle contraction has been tested in animal models. Argatroban was administered using the rat middle cerebral artery occlusion model. At 1 , 3 and $6 \mathrm{~h}$ following arterial occlusion, argatroban-treated animals showed significant reduction of secondary microthrombus formation in the brain (fig. 2) [8]. The inhibitory effect of argatroban on contraction of the isolated dog basilar artery was significant and showed a dose-dependent decrease in percentage rate of contraction following infusion of $0-10 \mathrm{U} / \mathrm{ml}$ of thrombin (fig. 3) [9].

Argatroban in Thrombotic Stroke 


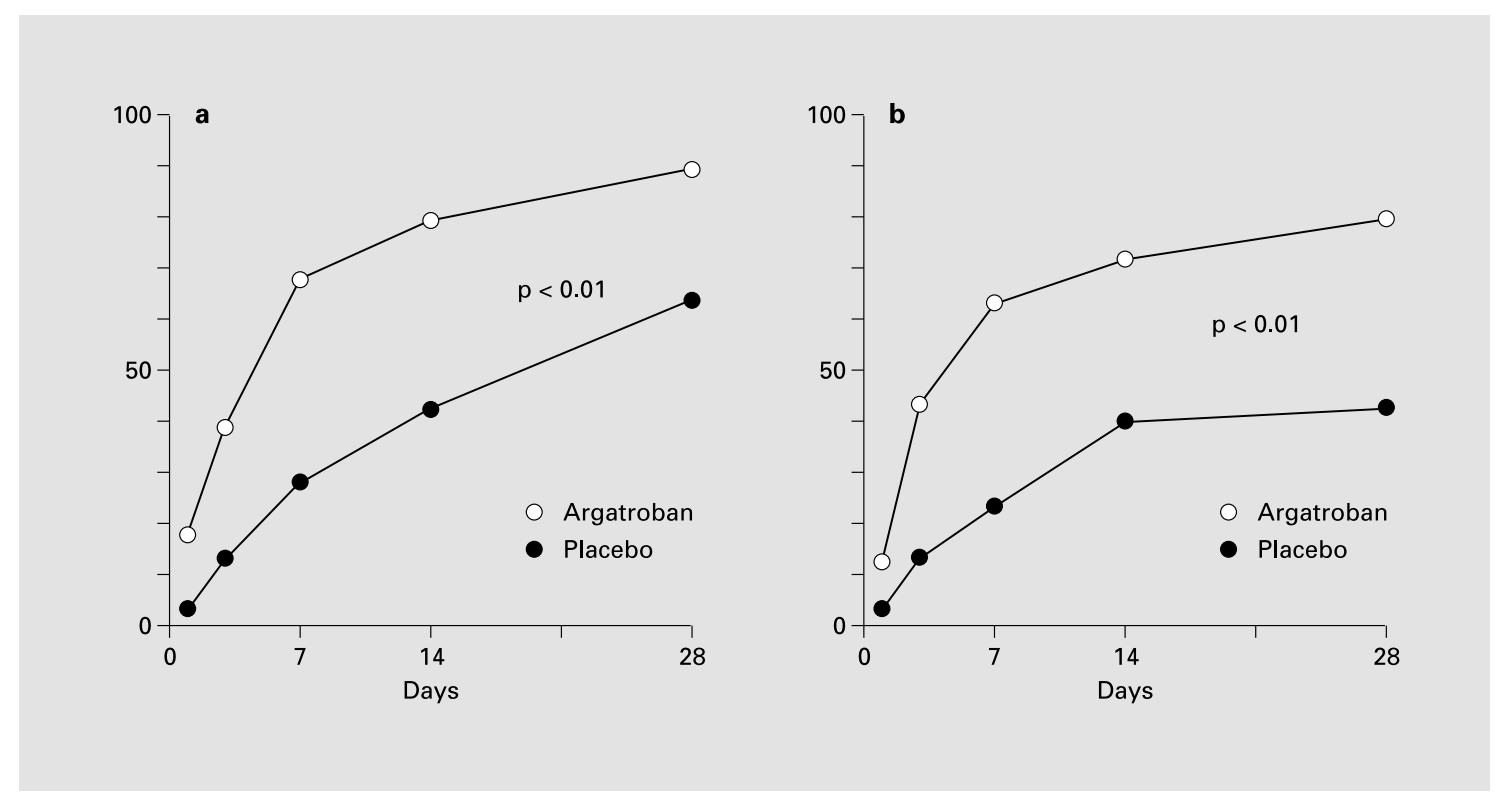

Fig. 4. Percentage of patients who showed more than one rank improvement. a ADL scale. b Paralyzed leg scale. From Kobayashi et al. [10].

Table 2. Global improvement rate of patients in the argatroban and placebo groups (from Kobayashi et al. [10])

\begin{tabular}{llll}
\hline & Total cases & \multicolumn{2}{l}{ Time from onset to administration } \\
\cline { 3 - 4 } & & within $48 \mathrm{~h}$ & over 48 h \\
\hline Argatroban & $54.2 \%(59)$ & $66.7 \%(30)$ & $41.4 \%(29)$ \\
Placebo & $23.7 \%(59)$ & $22.6 \%(31)$ & $25.0 \%(28)$ \\
Difference & $30.5 \%$ & $44.1 \%$ & $16.4 \%$ \\
& Wilcoxon test & Wilcoxon test & Wilcoxon test \\
& $(\mathrm{p}<0.01)$ & $(\mathrm{p}<0.01)$ & NS \\
\hline
\end{tabular}

Figures in parentheses are numbers of cases. Average aPTT $48 \mathrm{~h}$ after initiation of argatroban: 1.53 .

\section{Argatroban in Clinical Trials}

Some of the newer generation anticoagulants have been evaluated in advanced clinical trials of acute ischemic stroke. In Japan, argatroban has been tested in a multicenter, randomized, double-blind, placebo-controlled study of acute thrombotic stroke [10]. Fifty-two centers took part, recruiting a total of 119 patients. The protocol called for patients with thrombotic stroke to be randomized within 5 days of stroke onset to infusion of argatroban at $60 \mathrm{mg}$ per day continuously for $48 \mathrm{~h}$ fol- lowed by a 20 -mg intravenous infusion twice a day for 5 days versus placebo of sorbitol in a similar infusion. As a basic medication for patients in the acute stage, $500 \mathrm{ml}$ glycerol was administered once daily for 7 days.

Inclusion criteria were all patients with large artery atherothrombotic stroke diagnosed by clinical and laboratory evaluation including brain CT. Exclusion criteria were hemorrhagic stroke, lacunar stroke and patients suspected of cardioembolic ischemic stroke.

The primary outcome of the study was evaluation of global improvement on day 28 following initiation of therapy. Patient rating scales employed on days 1, 3, 7, 14 and 28 included the Japan Coma Scale, the Subjective Symptom Scale and the Activity Daily Living scale (ADL). Other neurological evaluations for motor, sensory, visual fields, dysarthria, dysphagia, aphasia, anosognosia and level of consciousness were carried out on the same days. Global improvement was judged by the site investigators on the basis of results of rating scales devised for consciousness, motor and sensory findings, psychological symptoms and specific activities of daily living.

The results showed that the mean global improvement of patients at 28 days was statistically significant for the group of patients treated with argatroban within $48 \mathrm{~h}$ of stroke symptom onset compared to that of the control patients (table 2). Overall, there was a 54.2\% mean improvement in the argatroban group of patients vs. 
Fig. 5. Brain Xe CT scans at baseline $(3 \mathrm{~h})$, 6 , and $12 \mathrm{~h}$ demonstrating hemispheric and penumbral $\mathrm{rCBF}$ of an ischemic stroke patient treated with argatroban.

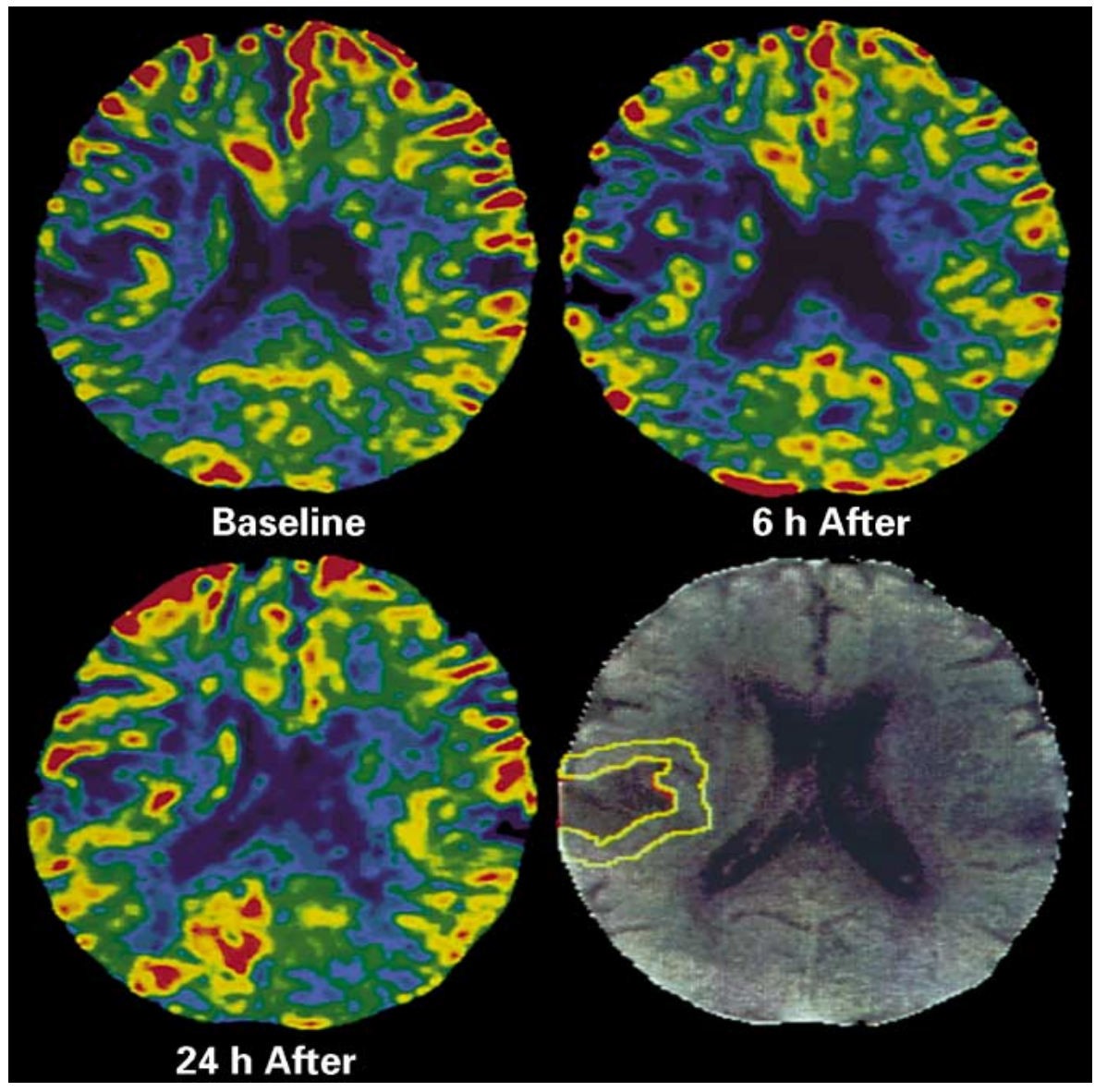

$23.7 \%$ in the placebo control group. Argatroban given later than $48 \mathrm{~h}$ after onset of symptoms produced a difference of $16 \%$ improvement over placebo (NS). Although levels of consciousness, subjective and psychological symptoms did not differ between the two groups, patients treated with argatroban showed statistically significant improvement compared with placebo for the ADL scale and the paralyzed leg scale (fig. 4). Brain CT scans showed that unchanged or decreased low-density areas were higher (but not significantly so) in the argatroban-treated group of patients. Adverse events reported in this study were low. Cerebral hemorrhage was detected in 1 of the 60 argatroban patients and in 2 of the 59 receiving placebo. All were minor and asymptomatic. After conclusion of this study, argatroban was approved for the treatment of thrombotic ischemic stroke when administered within $48 \mathrm{~h}$ of stroke symptom onset.

A further Japanese study involved patients with acute cerebral thrombosis, receiving argatroban [11]. This showed that by inhibiting thrombin activity, significant beneficial effects on regional cerebral blood flow occurred, postulated as due to protection of the penumbra. Furthermore, argatroban decreased FPA levels in these patients, indicating lower thrombin activity.

The effects of argatroban were further evaluated in a retrospective study of acute ischemic stroke patients. Subjects had xenon-CT regional cerebral blood flow (rCBF) scans at baseline and at prespecified time intervals following initiation of argatroban infusion therapy [12]. There was an increase in regional cerebral blood flow in the penumbra and in both hemispheres for both cortical and lacunar infarction when patients had scans performed at 3 , 6 and $24 \mathrm{~h}$ following onset of stroke symptoms (fig. 5, 6).

These investigations suggest that argatroban therapy in acute ischemic stroke patients has the potential to reduce secondary microthrombi in the penumbra, improve regional cerebral blood flow, reduce ischemic lesions and ameliorate neurological deficits.

Argatroban in Thrombotic Stroke 


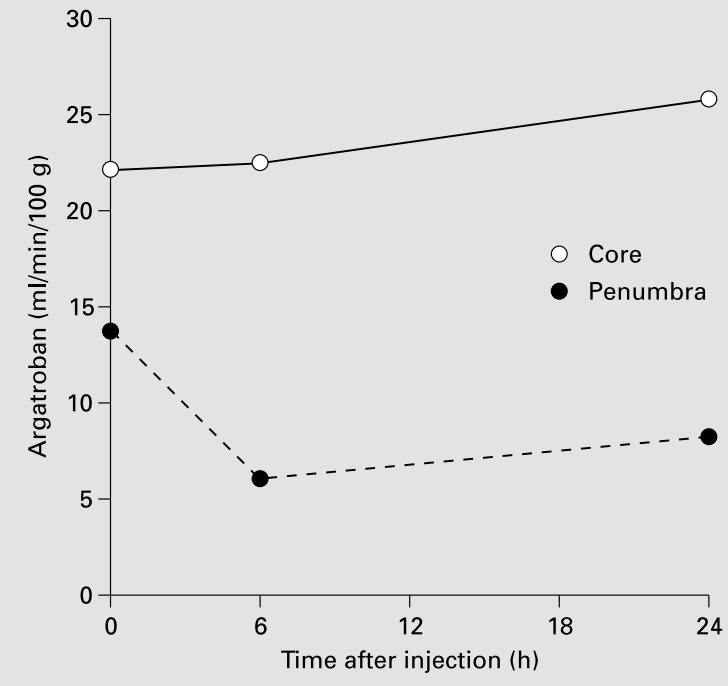

Fig. 6. Effect of argatroban on $\mathrm{rCBF}$.

Studies in the US investigating the benefit of argatroban in patients with heparin-induced thrombocytopenia with and without thrombosis syndrome (HIT, HITTS) found the direct thrombin inhibitor to reduce thrombosis events [13]. LaMonte et al. [14] reviewed the effects of argatroban therapy on neurologic complications in 1,005 HIT patients. Argatroban therapy, versus control, significantly reduced new stroke $(1.8 \%$ versus $3.1 \%, \mathrm{p}=0.032)$ in HIT [LaMonte MP, Brown PM, Hursting M, in preparation]. Intracranial hemorrhage was rare with one occurring in an argatroban-treated patient after therapy had ceased.

A safety study of argatroban in acute ischemic stroke is being conducted in the USA and Canada. The Argatroban in Acute Ischemic Stroke (ARGIS-1) study is a randomized, double-blind, placebo-controlled trial testing safety and exploring the efficacy of argatroban in patients with acute ischemic stroke [15]. 65 centers participated in recruitment of 176 patients. Patients were randomly assigned to placebo versus one of two groups of argatroban treatment to produce two targeted aPTTs of 1.75 versus 2.25. Inclusion criteria allowed for ischemic stroke patients with NIH Stroke Scale (NIHSS) scores in the range of 5-22 to be enrolled up to $12 \mathrm{~h}$ after stroke symptom onset.

The primary outcome of the study is safety as determined by symptomatic intracerebral hemorrhage and

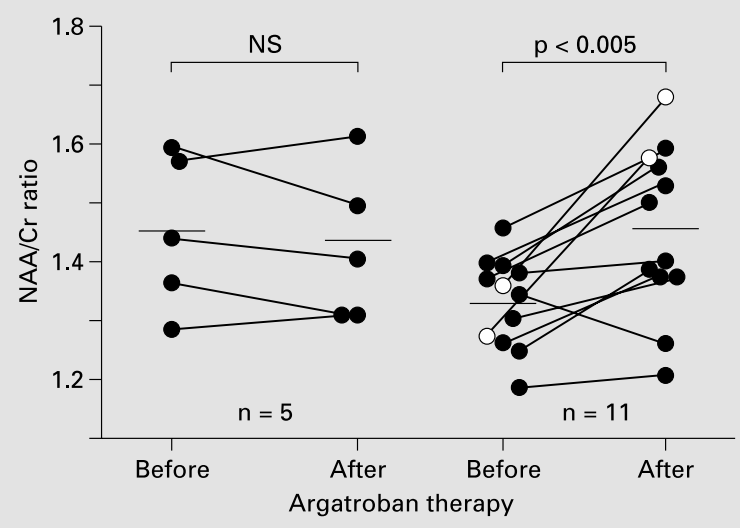

Fig. 7. $\mathrm{NNA} / \mathrm{Cr}$ ratio in deep white matter measured by MRS.

other major bleeding events. Secondary outcomes included further safety analyses and exploration of the efficacy of argatroban using the NIHSS score, and the modified Rankin and Barthel Index scales. The study has completed enrollment and results become available in 2003.

Cerebrovascular diseases other than acute ischemic stroke may be the targets for treatment in the future. For example, a small pilot study from Japan assessed the use of argatroban in patients with chronic cerebrovascular disease [16]. A total of 17 patients, 15 with silent cerebral infarction and 2 with vascular dementia participated. Patients were monitored before and after therapy for changes in neuronal activity, measured by spectroscopy MRI scan changes in NAA/creatine ratio in the deep white matter of the brain. Changes in coagulation activation markers were also measured. The results indicated that in the 11 patients with advanced cerebrovascular disease, the mean NAA/Cr ratio increased significantly from baseline, suggesting an improvement in neuronal activity (fig. 7). Among patients with less pronounced cerebrovascular disease, these differences were not significant. Overall changes in coagulation activation markers were statistically significant for TAT, showing a fall from baseline of 11.0 to 5.9 (table 3 ).
44

Pathophysiol Haemost Thromb 2002;32(suppl 3):39-45
LaMonte 
Table 3. Changes in coagulation markers in patients with cerebrovascular diseases

\begin{tabular}{|c|c|c|c|c|c|c|}
\hline & \multirow{2}{*}{\multicolumn{2}{|c|}{$\begin{array}{l}\text { Total patients } \\
(\mathrm{n}=17)\end{array}$}} & \multicolumn{4}{|c|}{ Cerebrovascular disease } \\
\hline & & & \multicolumn{2}{|c|}{ less $(n=5)$} & \multicolumn{2}{|c|}{ advanced $(n=12)$} \\
\hline & before & after & before & after & before & after \\
\hline $\mathrm{TAT}, \mathrm{ng} / \mathrm{ml}$ & 11 & $5.9^{\mathrm{a}}$ & 11.3 & 8.22 & 11.0 & $4.93^{\mathrm{b}, \mathrm{c}}$ \\
\hline $\mathrm{F} 1+2, \mathrm{nmol} / 1$ & 1.40 & $0.97^{\mathrm{b}}$ & 1.53 & $1.07^{b}$ & 1.35 & 0.94 \\
\hline $\mathrm{D}$ dimer, $\mathrm{mg} / \mathrm{dl}$ & 1.54 & 1.33 & 2.23 & 1.85 & 1.25 & 1.11 \\
\hline ATIII, \% & 93 & 102 & 97 & 103 & 92 & 101 \\
\hline
\end{tabular}

${ }^{\mathrm{a}} \mathrm{p}<0.01,{ }^{\mathrm{b}} \mathrm{p}<0.05$ versus before argatroban therapy.

\section{Conclusions}

There may be a role for thrombin inhibition in the treatment of cerebral ischemia. The mode of action of argatroban in cerebral thrombosis is explained as the inhibition of the local thrombin formed due to ischemic tissue damage. By preventing subsequent thrombus formation in the penumbral microcirculation it is hypothesized that improved blood flow to the peri-infarct penumbra would preserve neuronal cells at risk. In a clinical study of ischemic stroke in Japan, argatroban administered within $48 \mathrm{~h}$ of onset was found to safely and effectively reduce neurological deficit. A USA and Canadian study of argatroban's safety in acute ischemic stroke is ongoing. This multicenter, randomized, double-blind, placebo-controlled trial has a primary safety endpoint. Efficacy will be explored via a number of secondary outcomes.

\section{References}

1 Gubitz G, Counsell C, Sandercock P, Signrini D: Anticoagulants for acute ischaemic stroke (Cochrane Review). The Cochrane Library, Issue 2, 2001. Oxford: Update Software.

2 Collaborative group, CAST (Chinese Acute Stroke Trial): Randomized placebo-controlled trial of early aspirin use in 20,000 patients with acute ischemic stroke. Lancet 1997:349:16411649.

3 Chen ZM, Sandercock P, Pan HC, Collaborative group: The International Stroke Trial (IST): A randomized trial of aspirin, subcutaneous heparin, both, or neither among 19,435 patients with acute ischemic stroke. Lancet 1997;349:1569-1581.

4 Counsell C, Collins R, Liu LS, Xie JX, Warlaow $\mathrm{C}$, Peto R: Indications for early aspirin use in acute ischemic stroke: A combined analysis of 40,000 randomized patients from the Chinese acute stroke trial and the international stroke trial. On behalf of the CAST and IST collaborative groups. Stroke 2000;316:1240 1249.

5 Counsell C, Sandercock P: Low-molecularweight heparins or heparinoids versus standard unfractionated heparin for acute ischemic stroke (Cochrane Review). The Cochrane Library, Issue 2, 2001. Oxford: Update Software.
6 Uchiyama S, Yamazaki M, Hara Y, Iwata M: Alterations of platelet, coagulation and fibrinolysis markers in patients with acute ischemic stroke. Semin Thromb Haemost 1997;23:535541

7 Kario K, Komada K, Koide M, Matsuo T: Thrombin inhibition in the acute phase of ischaemic stroke using argatroban. Blood Coagul Fibrinolysis 1995;6:423-427.

8 Kawai K, Yuki S, Sugimoto J, Tamao Y: Effects of a thrombin inhibitor, argatroban, on ischemic brain damage in the rat distal middle cerebral artery occlusion model. J Pharmacol Exp Ther 1996;278:780-785.

9 Nakamura K: Thrombin-induced vasoconstriction in isolated cerebral arteries and the influence of a synthetic thrombin inhibitor. Thromb Res Suppl 1985;40:715-720.

10 Kobayashi S, Tazaki Y: Effect of the thrombin inhibitor argatroban in acute cerebral thrombosis. Semin Thromb Haemost 1997;23:531534.

11 Tanaka Y, Kawabata S, Sin R, Takatuka K, Nisimura H, Yoshikawa N, Komato T: Therapeutic effect of argatroban (MD-805) antithrombin agent in the acute stage of cerebral thrombosis. J Clin Ther Med 1987;3:133-142.
12 Maruki T, Onada A, Matsuzaki M Narabayashi Y, Sawada M, Shimazu K: A specific thrombin inhibitor (argatroban) ameliorated cerebral blood flow in the patients with acute cerebral infarction. Keio J Med 2000;49(suppl 1):138-140.

13 Lewis BE, Wallis DE, Berkowitz DS, Matthai WH, Fareed J, Walenga JM, Bartholomew J, Sham R, Lerner RG, Zeigler ZR, Rustagi PK, Jong IK, Rifkin SD, Moran J, Hursting MJ, Kelton JG for the ARG-911 Study Investigators: Argatroban anticoagulant therapy in patients with heparin induced thrombocytopenia. Circulation 2001;103:1838-1843.

14 LaMonte M, Brown P, Berens KL: Reduction in stroke mortality in patients with heparininduced thrombocytopenia after treatment with the direct thrombin inhibitor argatroban. Cerebrovasc Dis 2002;13(suppl 3):6.

15 LaMonte MP, Brown PM, Evans, DM: A randomized, placebo-controlled study to determine the safety and efficacy of argatroban injection in acute ischemic stroke (ARGIS I) (abstract). Cerebrovasc Dis 2002;13(suppl 3):6.

16 Kario K, Matsuo T, Shimada K: Department of Cardiology, Jichi Medical School, Department of IM. Hygo Prefectural Awaji Hospital, unpublished data. 